\title{
Epstein-Barr virus-positive follicular lymphoma
}

Nicholas Mackrides ${ }^{1}$, German Campuzano-Zuluaga ${ }^{1}$, Yvan Maque-Acosta ${ }^{1}$, Adrienne Moul ${ }^{1}$, Nouf Hijazi ${ }^{1}$, Francis Offiong Ikpatt ${ }^{1}$, Ronald Levy ${ }^{2}$, Ramiro E Verdun ${ }^{3}$, Kranthi Kunkalla ${ }^{1}$, Yasodha Natkunam ${ }^{4}$, Izidore S Lossos ${ }^{5}$, Francisco Vega ${ }^{1,5}$ and Jennifer Chapman ${ }^{1}$

${ }^{1}$ Division of Hematopathology, Department of Pathology and Laboratory Medicine, University of Miami and Sylvester Comprehensive Cancer Center, Miami, FL, USA; ${ }^{2}$ Division of Oncology, Department of Medicine, Stanford University, Stanford, CA, USA; ${ }^{3}$ Department of Medicine, Division of Hematology-Oncology, Sylvester Comprehensive Cancer Center. University of Miami, Miami, FL and GRECC, Miami VAHS, FL, USA; ${ }^{4}$ Department of Pathology, Stanford University, Stanford, CA, USA and ${ }^{5}$ Division of Hematology-Oncology, Department of Medicine, University of Miami and Sylvester Comprehensive Cancer Center, Miami, FL, USA

Epstein-Barr virus (EBV) -associated follicular lymphoma is only rarely reported. Herein, we report the largest series analyzing prevalence and clinicopathologic characteristics of EBV-associated follicular lymphoma occurring in unselected cases. Out of 382 analyzed cases, 10 EBV-positive follicular lymphomas were identified (prevalence $=2.6 \%, 95 \%$ confidence interval 1.3-4.0\%). All EBV-positive follicular lymphomas showed EBVencoded small RNA-positive lymphoma cells present in a follicular distribution. Of these, eight also had tissue available for testing of expression of latent membrane protein 1 (LMP1), out of which six (75\%) were positive. There was a significant association with grades 3A-3B follicular lymphoma $(P<0.0001)$ and CD30 expression $(P=0.0002)$. EBV-positive follicular lymphomas were otherwise morphologically and immunophenotypically indistinguishable from EBV-negative cases of similar grade. Nine of the EBV-positive follicular lymphomas occurred in patients with no known history of immunosuppression, while one patient had a history of hydroxychloroquine administration for Sjögren's syndrome. The mean age in the EBV-positive and -negative follicular lymphomas was 56 (range 31-83 years) and 49 years (range 25-92 years), respectively, with no statistically significant difference. Seven of the patients with EBV-positive follicular lymphoma had additional biopsies from different time points available for review, all of which showed progression of disease in the form of progression of tumor grade. Five of these progressed to diffuse large B-cell lymphoma, one of which had tissue available for testing and was EBV-positive. Our findings suggest that EBV infection may have a role in lymphomagenesis and/or disease progression in a subset of follicular lymphomas, thereby expanding the spectrum of recognized EBV-associated B-cell lymphomas.

Modern Pathology (2017) 30, 519-529; doi:10.1038/modpathol.2016.214; published online 16 December 2016

Epstein-Barr virus (EBV), a member of the Herpesviridae family, also called human herpesvirus 4 (HHV$-4)$, is one of the most common viruses that infects humans and is characterized by the property of latency. ${ }^{1} \mathrm{EBV}$ is associated with the development of several types of lymphomas, particularly in patients with congenital or acquired immunodeficiency. ${ }^{2}$

Correspondence: Dr J Chapman, MD, Division of Hematopathology, Department of Pathology and Laboratory Medicine, University of Miami Hospital and Sylvester Comprehensive Cancer Center, 1400 NW 12th Avenue, 4th Floor, Room 4076, Miami, FL 33138, USA.

E-mail: jchapman@med.miami.edu

This paper was presented in part at the United States and Canadian Academy of Pathology (USCAP) Annual meeting, Seattle (WA), 2016.

Received 8 August 2016; revised 22 October 2016; accepted 24 October 2016; published online 16 December 2016
Most EBV-related lymphomas are of a B-cell lineage; however, a subset of NK and T-cell lymphomas are also associated with EBV. ${ }^{3,4}$ Specifically recognized B-cell lymphomas associated with EBV include classical Hodgkin lymphoma, Burkitt lymphoma, lymphomatoid granulomatosis, post transplant and other lymphoproliferative disorders associated with immunodeficiency, and subsets of diffuse large B-cell lymphoma. Large B-cell lymphomas associated with EBV are usually aggressive neoplasms and include several subtypes: plasmablastic lymphoma, diffuse large B-cell lymphoma associated with chronic inflammation, primary central nervous system lymphoma arising in immunosuppressed patients, and EBV-positive diffuse large B-cell lymphoma. ${ }^{5}$

Recently, during the course of our clinical practice, we observed two cases of grade $3 \mathrm{~A}$ follicular 
lymphoma, one of which had a component of diffuse large B-cell lymphoma, which were found to be EBV-associated based on positive expression of EBVencoded small RNA (EBER) as determined by in situ hybridization. EBER testing in the first of these two cases was performed based on the patient's advanced age of 86 years and the presence of a high-grade (3A) follicular lymphoma with a diffuse large B-cell lymphoma component. In this case, EBER was strongly positive in lymphoma cells present in both the diffuse and follicular components. Lymphoma cells in this case were also positive for LMP1. This patient had a previous history of high-grade follicular lymphoma, grade $3 \mathrm{~A}$, based on an excisional biopsy in which a diffuse large B-cell lymphoma component was not present. EBER testing was performed retrospectively in this patient's initial biopsy and was positive in the follicular lymphoma despite the absence of a diffuse large B-cell lymphoma component at that time. Shortly after this case, a second case of EBV-positive grade 3A follicular lymphoma without a diffuse large B-cell lymphoma component was seen in a 76-year-old patient who had a previous history of low-grade follicular lymphoma for 6 years.

Currently, the association between EBV and typical follicular lymphoma occurring in the setting of immunocompetence is not well recognized. Only few sporadic cases of EBV-positive follicular lymphoma have been documented in the literature. ${ }^{6-8}$ Most of the previously reported cases were in the form of isolated case reports identified in patients with significant immunodeficiency and usually in the context of transformation to high-grade lymphomas and/or classical Hodgkin lymphomas. ${ }^{6-8}$ Because EBER is not systematically assessed in follicular lymphoma, the prevalence, underlying biology, clinicopathological features and prognostic significance of EBV-positive follicular lymphoma is unknown. On the basis of our initial findings of two EBER-positive follicular lymphoma cases in elderly but otherwise immunocompetent patients, we decided to investigate the incidence of EBVassociated follicular lymphoma on a larger scale. To this end we analyzed 382 cases of unselected follicular lymphoma for the presence of EBV in order to provide an estimated prevalence of this disease and assess if EBV-positive cases have distinctive clinicopathologic features in comparison to EBV-negative follicular lymphomas.

\section{Materials and methods}

We identified 382 cases of follicular lymphoma occurring in adults and consecutively diagnosed between 2011 and 2015 at the University of Miami and between 1985 and 2000 at Stanford University for which formalin fixed paraffin-embedded tissue was available for testing with EBER in the form of a tissue microarray or full tissue sections. Cases contributed from the University of Miami were reviewed as whole tissue sections from diagnostic patient samples. The tissue microarray used in this study was made from follicular lymphoma cases seen by one physician at Stanford University (RL) over 19 years. Only cases with in-house formalin fixed paraffin-embedded blocks with sufficient material for duplicate $1.0 \mathrm{~mm}$ cores to make the tissue microarray were included. Testing with LMP1 was also performed in cases in which sufficient tissue was available. Tissue microarray sections were created from representative areas of complete tissue sections in which the interpretation and diagnosis of follicular lymphoma was made in 343 out of the 382 cases; 39 cases were reviewed as whole tissue sections. Cases of diffuse large B-cell lymphoma, primary cutaneous or primary gastrointestinal (duodenal-type) follicular lymphoma, and pediatric-type follicular lymphoma were not included in this study. Clinical and pathology information was obtained by retrospective review of the medical chart and pathology reports after institutional review board approval. The diagnosis and grading of all lymphomas was based on criteria as specified in the 2008 WHO classification and all cases were reviewed and diagnosed by hematopathologists at either the University of Miami or Stanford University.

Immunohistochemical studies were performed on formalin fixed paraffin-embedded tissue sections on Leica BOND-III automated stainers and included a variable panel of B- and T-cell markers used to support the diagnosis of follicular lymphoma. Evaluated immunohistochemistry markers included CD20, CD3, CD10, BCL6, BCL2, LMP1, and CD30 (Leica Biosystems, Newcastle, UK). CD30 was considered positive when $>30 \%$ of lymphoma cells were positive.

Testing for the presence of EBV was performed by in situ hybridization for EBER on formalin fixed paraffin-embedded tissue sections on a Leica BOND-III automated stainer (Leica Biosystems). Appropriate positive and negative controls were used. EBV-positive cases were defined as those with greater than 10 EBER-positive tumor cell nuclei per $0.5 \mathrm{~cm}^{2}$ (ref 9). Cases with 'bystander' EBER-positive non-neoplastic lymphocytes were not included as EBV-positive follicular lymphoma cases.

Double immunofluorescence labeling was performed using formalin fixed paraffin-embedded tissue sections. Immunofluorescence for LMP1 was performed in combination with CD20 (LMP1 and CD20), BCL2 (LMP1 and BCL2) and BCL6 (LMP1 and BCL6). The slides were incubated with anti-LMP1 antibody (Dako, USA), and with an anti-CD20, BCL2, or BCL6 antibody (Abcam, USA) for $2 \mathrm{~h}$ at room temperature. Alexa Fluor 594 (red) goat anti-rabbit IgG conjugate and Alexa Fluor 488 (green) goat antimouse IgG conjugate antibody (Life Technologies, USA) were used as secondary antibodies. The nuclei were counterstained with 40-60 diamidino-2phenylindole (DAPI). After aqueous mounting, the 
Table 1 Immunohistochemistry and in situ hybridization in cases of EBV-positive follicular lymphoma

\begin{tabular}{|c|c|c|c|c|c|c|c|c|}
\hline Case & CD20 & CD30 (>30\%) & CD10 & $B C L 6$ & $B C L 2$ & FISH t(14;18) & $L M P 1$ & EBER \\
\hline 1 & + & + & + & ND & + & + & ND & + \\
\hline 2 & + & + & + & + & + & - & ND & + \\
\hline 3 & + & - & + & + & + & ND & + & + \\
\hline 4 & + & + & + & - & + & ND & + & + \\
\hline 5 & + & - & + & - & + & ND & + & + \\
\hline 6 & + & - & + & + & + & ND & - & + \\
\hline 7 & + & - & + & + & + & ND & - & + \\
\hline 8 & + & - & + & + & + & ND & + & + \\
\hline 9 & + & - & + & - & + & ND & + & + \\
\hline 10 & + & + & + & ND & + & ND & + & + \\
\hline
\end{tabular}

+, positive; -, negative; ND, not done.

slides were observed using a fluorescence microscope (Olympus BX51, Tokyo, Japan).

The presence of $\mathrm{t}(14 ; 18)(\mathrm{q} 32 ; \mathrm{q} 21) /$ IGH-BCL2 was investigated using interphase fluorescence in situ hybridization with a IGH/BCL2 dual-color, dual-fusion translocation probe (Vysis-Abbott, Des Plaines, Ill) on formalin fixed paraffin-embedded tissue sections in select cases in which sufficient tissue was available.

Statistical analysis included comparison of the number of EBV-positive cases among grade 1-2 and grade 3A-3B follicular lymphomas, and of CD30 expression, using Fisher exact test. All 95\% confidence intervals (CIs) were calculated via a basic bootstrap procedure using 2000 repeats. Comparison of patient age in EBV-positive versus EBV-negative follicular lymphomas was performed using the Wilcox rank-sum test. The significance level was set at an $\alpha$-value of 0.05 . $\mathrm{R}$ (version 3.1.3, http:// www.r-project.org) software was used for all statistical analyses.

\section{Results}

Out of the 382 identified cases of follicular lymphoma, each case represented an individual patient and all cases had sufficient diagnostic material available for pathology assessment and lymphoma diagnosis including morphologic and immunophenotypic interpretation, tumor grading, and EBER testing. All cases were initially reviewed as complete tissue sections in which the diagnosis of follicular lymphoma and tumor grade was confirmed by hematopathologists (YN, JC, and FV). Each case had morphological and immunophenotypic features typical for follicular lymphoma including follicular or follicular and diffuse pattern, morphological features of centroblasts and centrocytes within neoplastic follicles, and germinal center immunophenotype. Cases with abnormal large cells with significant anaplastic or Hodgkin-like features were not seen in this series. Tumor grade was available in all cases. Overall in this cohort, grade 1-2 follicular lymphoma represented $74.1 \%$ and grade $3 \mathrm{~A}-3 \mathrm{~B}$,
$25.9 \%$. The mean patient age was 49 years (range 25-92 years).

EBER in situ hybridization was performed in all cases, regardless of tumor grade, patient age or clinical stage and identified 10 EBV-positive follicular lymphomas (prevalence $=2.6 \%, 95 \%$ CI $1.3-4.0 \%$ ). Immunophenotypic findings for the 10 EBV-positive follicular lymphoma cases are provided in Table 1. Out of the 10 EBER-positive follicular lymphoma cases, all were composed of lymphoma cells present in a follicular distribution which expressed CD10 and coexpressed BCL2. Most of these cases also expressed BCL6 (Table 1). Tissue was available in two of the EBER-positive follicular lymphoma cases for interphase fluorescent in situ hybridization for translocation $\mathrm{t}(14 ; 18)(\mathrm{q} 32 ; \mathrm{q} 21) /$ IgH;BCL2, which was positive in one case and negative in one case. All EBV-positive follicular lymphoma cases showed strong labeling of EBER restricted to a follicular distribution that corresponded to that of the neoplastic follicles (Figures $1,2,3,4)$. Although 9 out of the 10 (90\%) EBV-positive follicular lymphoma cases showed EBER positivity present in $>75 \%$ of tumor cells within neoplastic follicles (Figures 1,2,4), one case of grade 3A follicular lymphoma showed EBER positivity in only $\sim 5-10 \%$ of lymphoma cells (Figure 3 ). This particular case had fluorescent in situ hybridization results available and was positive for $\mathrm{t}(14 ; 18) / \operatorname{IgH}$; BCL2. In cases of grade 3A-3B follicular lymphoma, EBV was present predominantly in large centroblasts (Figure 4). LMP1 expression was assessed in eight of the 10 EBV-positive cases and was positive in six of these $(75 \%)$, with overlapping of the distribution of the EBER-positive cells in each of these cases (Figure 4). Compared with EBER expression, LMP1 was identified in only a subset of follicular lymphoma cells. In a select case in which sufficient tissue was available for testing, double immunofluorescence staining confirmed that a subset of CD20-positive lymphoma cells were positive for LMP1 and that these same LMP1-positive, CD20positive cells coexpressed BCL6 and BCL2 (Figure 5). These findings support that the LMP1positive B-cells are neoplastic germinal center- 

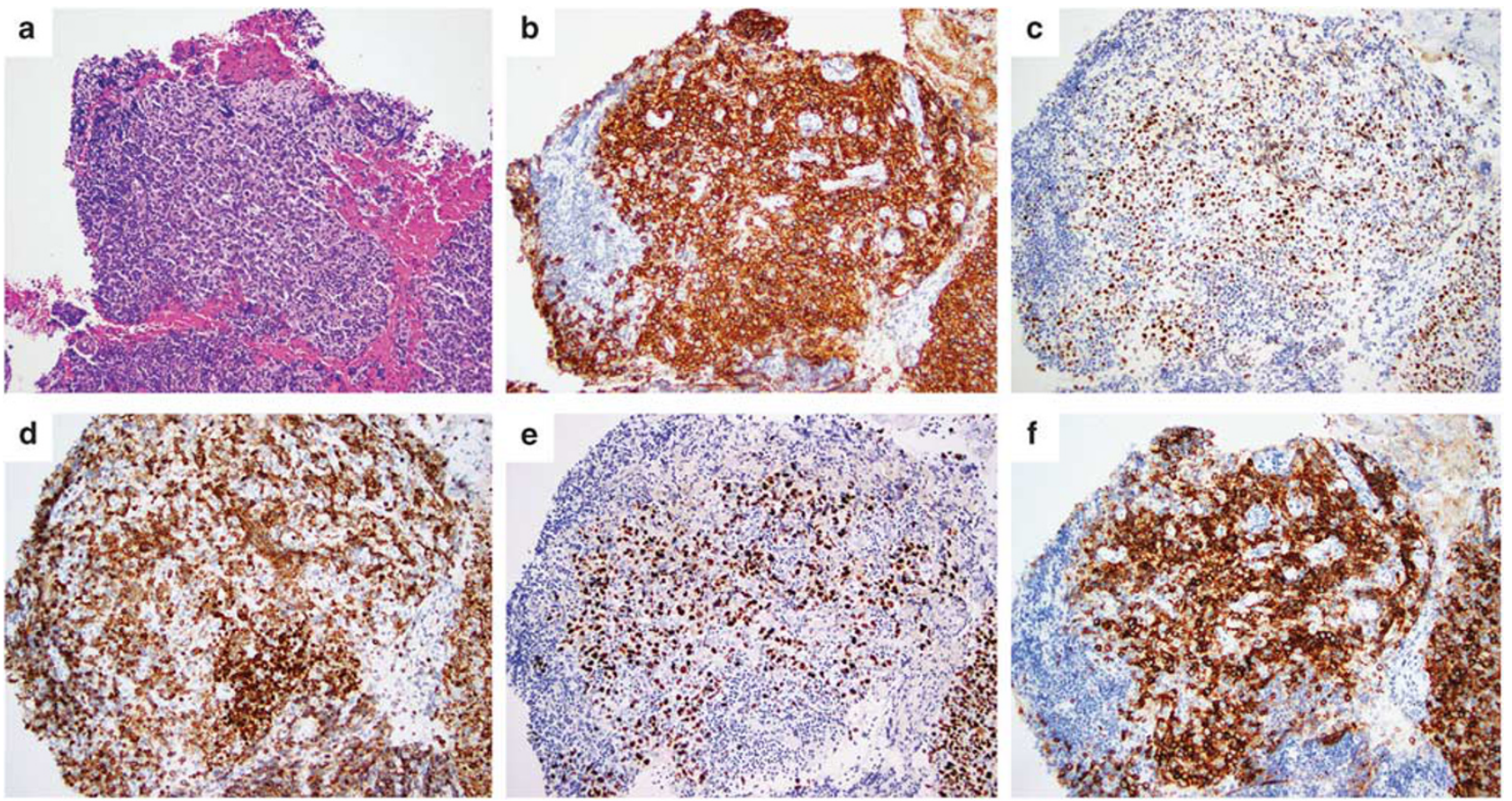

Figure 1 Follicular lymphoma, grade 3A, follicular pattern, CD30-positive, EBER-positive. Lymphoma cells are present in a follicular distribution and include increased large cells as seen on the H\&E stain (a). Lymphoma cells are positive for CD20 (b), BCL6 (c), and BCL2 (d). EBER was detected in $>75 \%$ of lymphoma cells in a follicular distribution (e). CD30 immunohistochemistry stain showing strongest expression in lymphoma cells within follicles with a distribution similar to that of EBER expression (f) (patient 2, Table 2). All images taken at $\times 200$.
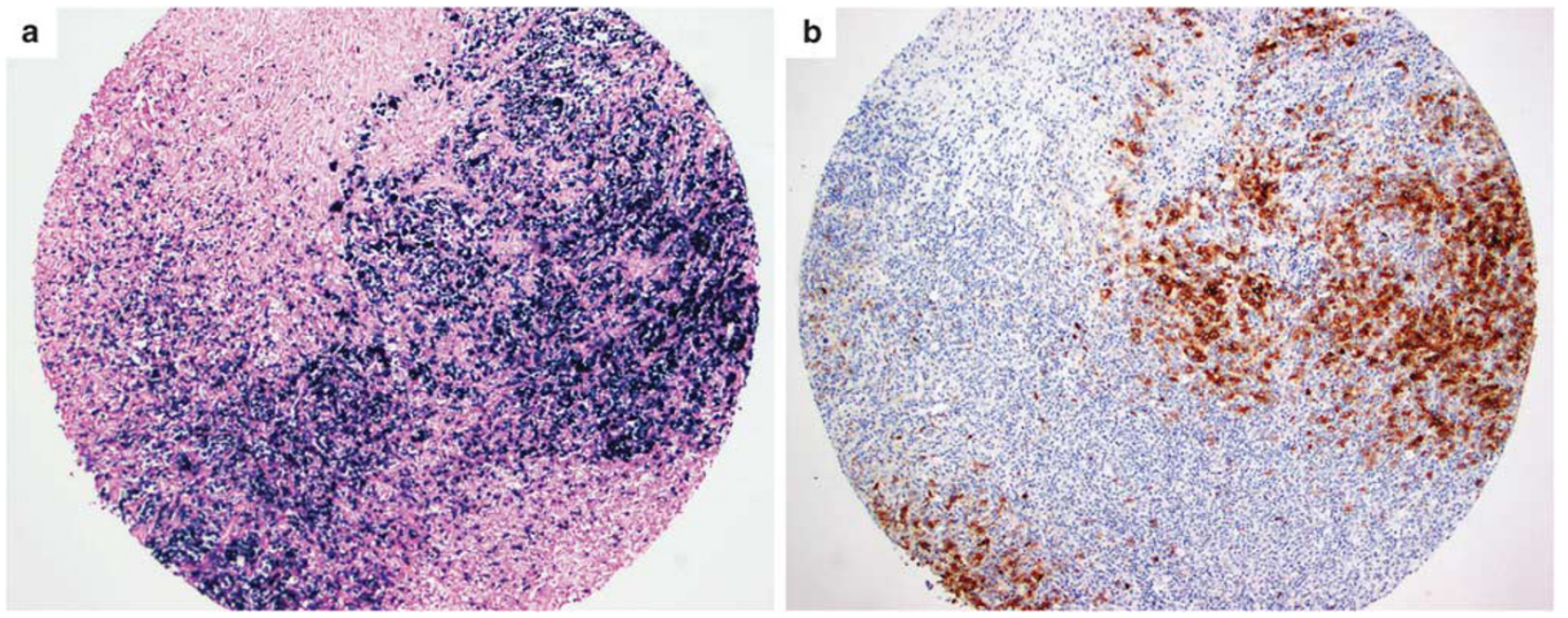

Figure 2 Follicular lymphoma, grade 3A, follicular pattern, CD30-positive, EBER-positive. EBER was detected in > 75\% of lymphoma cells in a follicular distribution. (a) CD30 immunohistochemistry stain shows strongest expression in lymphoma cells within follicles with a distribution similar to that of EBER expression (b). Images taken at $\times 200$.

derived B-cells and therefore support that LMP1 is present in follicular lymphoma cells.

Each of the 10 identified EBV-positive follicular lymphomas represented an individual patient biopsy identified at a single time point in their disease course. Nine $(90 \%)$ of the EBV-positive follicular lymphomas were grade 3A-3B compared with only one which was a grade 1-2 follicular lymphoma $(10 \%$ and $0.4 \%$ respectively, proportion difference: $9.6 \% ; 95 \%$ CI $4.7-14.6 \%$, Fisher's exact test $P$ value $<0.0001)$. Out of the nine EBV-positive grade 3A-3B follicular lymphoma cases, eight were grade $3 \mathrm{~A}$, and one was grade $3 \mathrm{~B}$. Clinical staging information was available in seven patients; four were stage 

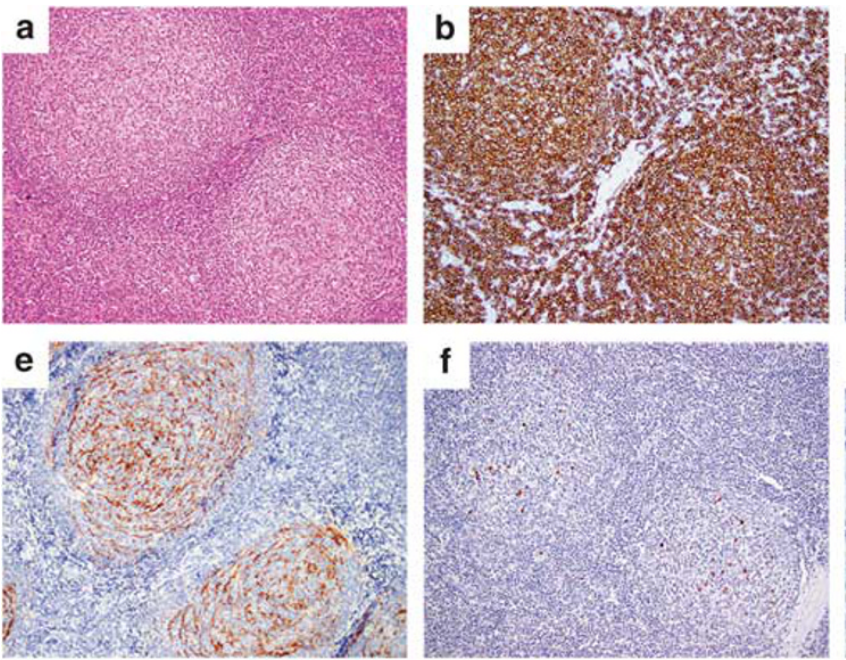
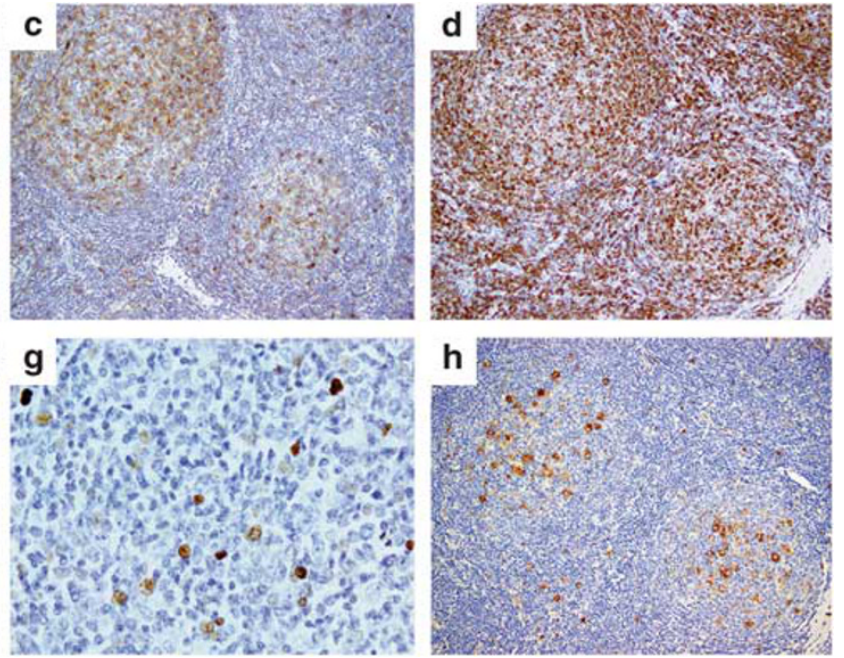

Figure 3 Follicular lymphoma, grade 3A, follicular pattern, CD30-positive, EBER-positive. Lymphoma cells are present in a follicular distribution and include increased ( $>15$ ) large centroblasts per high-power field as seen on the H\&E stain (a). Lymphoma cells coexpress CD20 (b), CD10 (c), and BCL2 (d). CD21 immunohistochemistry stain highlights the presence of follicular dendritic cell networks within neoplastic follicles (e). Positivity for EBER was seen in $~ 5-10 \%$ of lymphoma cells in a follicular distribution (f, g). CD30 immunohistochemistry stain shows strongest expression in lymphoma cells within follicles with a distribution similar to that of EBER expression (h). Fluorescent in situ hybridization for t(14;18)(IgH-BCL2) was positive in this case (patient 1, Table 2). All images taken at $\times 200$ except image $\mathrm{G}$, taken at $\times 500$ magnification.
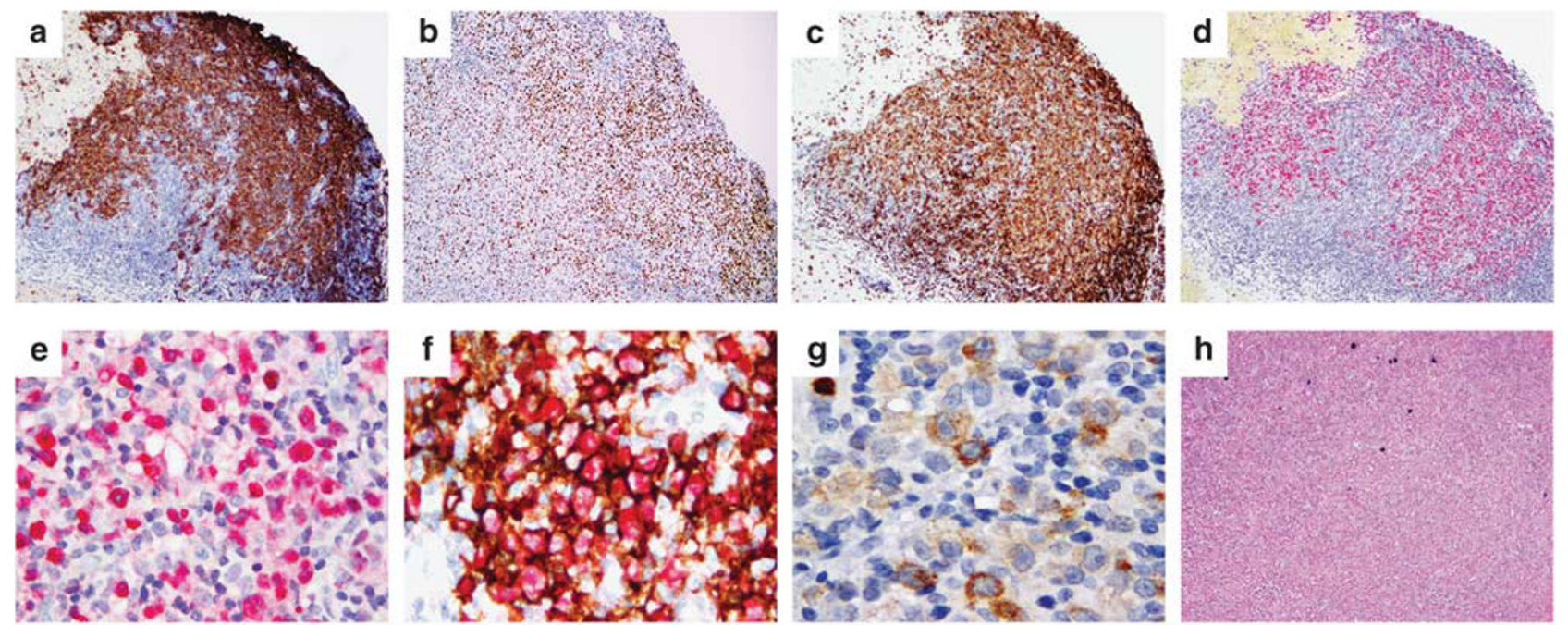

Figure 4 Follicular lymphoma, grade 3A, follicular pattern. Lymphoma cells are present in a follicular distribution and coexpress CD20 (a), BCL6 (b), and BCL2 (c) and are positive for EBV with a type II or III latency pattern as demonstrated by EBER positivity by in situ hybridization (d, e), dual staining with EBER (nuclear stain in red) and CD20 (membranous and cytoplasmic in brown) (f), and LMP1 positivity by immunohistochemistry (g) (patient 3, Table 2). An example of a FL which was negative for EBER is included as h. In this example, scattered EBER-positive cells are seen but these are interfollicular and do not correspond in location or morphology to those of the lymphoma cells. Images $\mathbf{a}-\mathbf{d}$ and $\mathbf{h}$ were taken at $\times 200$, images E-G were taken at $\times 500$.

IV, two stage III, and one stage II. Detailed clinical information was available for seven out of the 10 EBV-positive cases and is provided in Table 2. None of the included patients were known to be HIV positive. HIV information was captured from the lymphoma database of Stanford University or was based on individual patient testing at the University of Miami (tested in two out of the three patients). Aside from possible age-related immunosenescence, six of the patients were otherwise immunocompetent. One patient had a history of long-term treatment with hydroxychloroquine for Sjögren's syndrome. In this single case, because the EBV-positive follicular lymphoma was occurring in the context of treatment with an immunomodulator, this lymphoma could be classified as an 

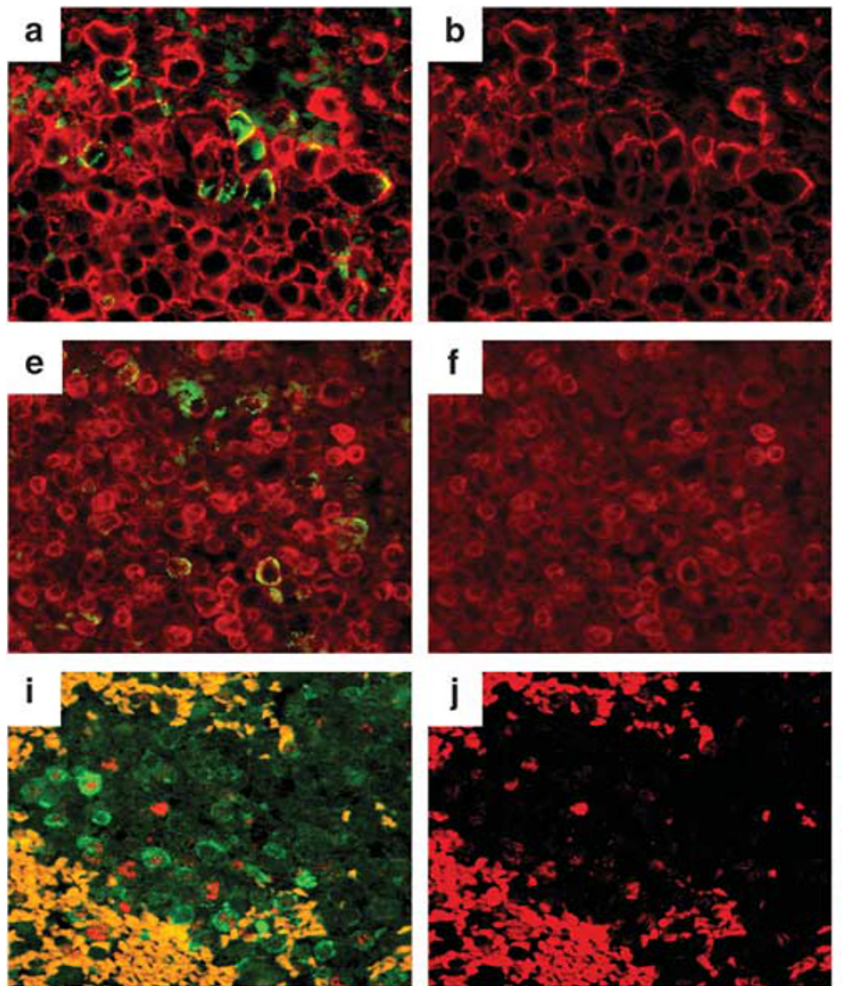
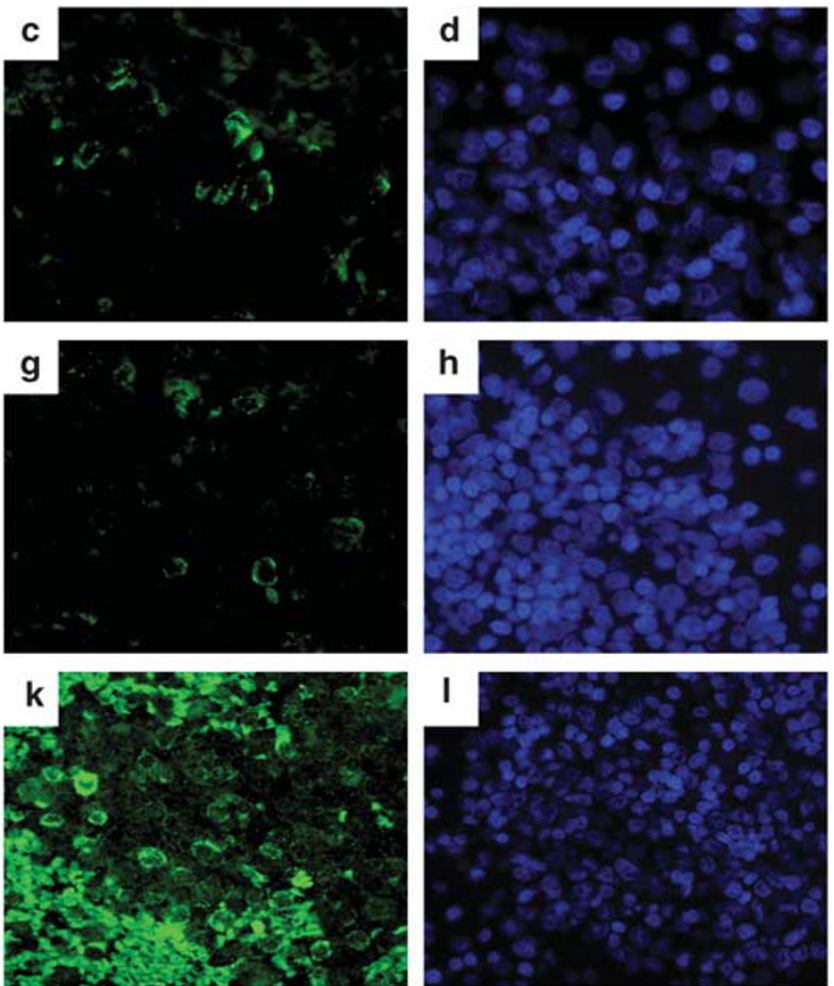

Figure 5 Dual immunofluorescence staining for CD20, BCL2, and BCL6 with LMP1 in EBV-positive FL. Double immunofluorescence analysis shows that the LMP1-positive cells are positive for CD20 (a), BCL2 (e) and BCL6 (i) supporting that they are follicular lymphoma cells. CD20 (a and b), BCL2 (e and f), and BCL6 (i and j) stains are shown labeled in red and LMP1 is labeled in green (c, g, and $\mathbf{k})$ (d, h, and $\mathbf{j}$ ). DAPI was used to counterstain the nuclei (last image in each lane).

iatrogenic immunodeficiency-associated lymphoproliferative disorder. This patient was not treated with methotrexate or any of the other agents more commonly associated with iatrogenic immunodeficiency-related lymphoma, however. Seven out of the 10 patients with EBV-positive follicular lymphoma had additional biopsies from different time points during their disease course; two of these patients had prior biopsies (samples that were taken at a time point before the one included in this cohort) and five had subsequent biopsies (taken at a time point subsequent to the one included in this cohort). Both patients with previous biopsies had EBV-positive grade 3A follicular lymphoma at the time of inclusion in this cohort and both had previous histories of grade 1-2 disease. Biopsy material was not available from the previous grade 1-2 follicular lymphomas, thus EBV status is unknown at the time of their lowgrade disease (patients 2 and 4, Table 2). Out of the five patients with subsequent biopsies, one had EBVpositive grade 1-2 follicular lymphoma at the time of inclusion in this cohort that progressed to diffuse large B-cell lymphoma (patient 5) and the others had EBV-positive grade $3 \mathrm{~A}$ follicular lymphoma that progressed to diffuse large B-cell lymphoma (patients 1, 7, 8, 10). Biopsy material from one of the latter was available at both time points and both the grade $3 \mathrm{~A}$ follicular lymphoma and diffuse large B-cell lymphoma were EBER-positive. Thus, the seven patients with biopsies available at separate time points all demonstrated progression of disease; two from grade 1-2 follicular lymphoma to grade 3A, one from grade 1-2 follicular lymphoma to diffuse large B-cell lymphoma, and four from grade $3 \mathrm{~A}$ to diffuse large B-cell lymphoma (Table 2).

Immunohistochemistry staining for CD30 was performed on 241 cases. Overall, CD30 expression was found in nine cases of follicular lymphoma (3.7\% of cases tested). In CD30-positive follicular lymphoma cases, CD30 expression was present in a follicular distribution corresponding to neoplastic lymphoma cells within follicles (Figures 1,2,3). CD30 expression was significantly more frequent in EBV-positive follicular lymphoma compared with EBV-negative follicular lymphoma (40 versus $2.2 \%$; proportion difference: $37.8 \%$, 95\% CI: $10.4-65.3 \%$; Fisher's exact test $P$-value $=0.0002$ ). CD30-positive, EBV-positive follicular lymphomas were otherwise morphologically and immunophenotypically indistinguishable from EBV-negative and CD30-negative follicular lymphomas of similar grade. The mean age in the EBER-positive and -negative groups was 56 (range 31-83 years) and 49 (range 25-92 years) years, respectively, with no statistically significant difference among these groups ( $P$-value 0.1796). In addition to and separate from the cases of 


\begin{tabular}{|c|c|c|c|c|c|c|c|c|c|c|c|}
\hline Case & $\begin{array}{c}\text { Age } \\
\text { (years) }\end{array}$ & Clinical presentation & Immunosuppression & $\begin{array}{l}\text { Initial } \\
F L \\
\text { grade }\end{array}$ & $\begin{array}{l}\text { Stage at } \\
\text { initial } \\
\text { diagnosis }\end{array}$ & $\begin{array}{l}\text { Initial } \\
\text { treatment }\end{array}$ & Progression & $\begin{array}{l}\text { Time from initial } \\
\text { diagnosis to } \\
\text { progression (years) }\end{array}$ & $\begin{array}{l}\text { Treatment at } \\
\text { disease } \\
\text { progression }\end{array}$ & $\begin{array}{l}\text { Alive/deceased } \\
\text { at last follow-up }\end{array}$ & $\begin{array}{l}\text { Follow-up time } \\
\text { from initial } \\
\text { diagnosis (years) }\end{array}$ \\
\hline 1 & 83 & $\begin{array}{l}\text { Cervical lymphadeno- } \\
\text { pathy }\end{array}$ & No & $3 \mathrm{~A}$ & IVA & $\begin{array}{l}\text { Expectant } \\
\text { followed by } \\
\text { Rituximab }\end{array}$ & $\begin{array}{l}\text { Yes } \\
\text { Grade 3A to } \\
\text { DLBCL }\end{array}$ & 3.8 & R-CHOP & DOD & 4.2 \\
\hline 2 & 76 & $\begin{array}{l}\text { Abdominal } \\
\text { lymphadenopathy }\end{array}$ & $\begin{array}{l}\text { Hydroxychloroquine } \\
\text { for Sjögren's Syndrome }\end{array}$ & $1-2$ & IVA & None & $\begin{array}{l}\text { Yes } \\
\text { Grade 1-2 to } \\
3 \mathrm{~A}\end{array}$ & 6.1 & Rituximab & DOD & 14 \\
\hline 3 & 60 & $\begin{array}{l}\text { Cervical and inguinal } \\
\text { lymphadenopathy }\end{array}$ & No & 3B & $\mathrm{U}$ & $\mathrm{U}$ & $\mathrm{U}$ & NA & $\mathrm{U}$ & Alive & 0.1 \\
\hline 4 & 33 & $\begin{array}{l}\text { Cervical } \\
\text { lymphadenopathy }\end{array}$ & No & $1-2$ & IVA & None & $\begin{array}{l}\text { Yes } \\
\text { Grade 1-2 to } \\
3 \mathrm{~A}\end{array}$ & 4.3 & CVP & Alive & 11.8 \\
\hline 5 & 48 & $\begin{array}{l}\text { Inguinal } \\
\text { lymphadenopathy }\end{array}$ & No & $1-2$ & IIIA & None & $\begin{array}{l}\text { Yes } \\
\text { Grade 1-2 to } \\
\text { DLBCL }\end{array}$ & 7.2 & $\begin{array}{l}\text { CVP and } \\
\text { fludarabine, } \\
\text { idiotype } \\
\text { vaccine, KLH, } \\
\text { autologous } \\
\text { BMT }\end{array}$ & Alive & 17.6 \\
\hline 6 & 76 & $\begin{array}{l}\text { Enlarged left inguinal } \\
\text { lymph node }\end{array}$ & No & $3 \mathrm{~A}$ & $\mathrm{U}$ & $\mathrm{U}$ & $\mathrm{U}$ & NA & $\mathrm{U}$ & $\mathrm{U}$ & 0 \\
\hline 7 & 31 & $\begin{array}{l}\text { Mediastinal } \\
\text { lymphadenopathy }\end{array}$ & No & $3 \mathrm{~A}$ & IVA & None & $\begin{array}{l}\text { Yes } \\
\text { Grade 3A to } \\
\text { DLBCL }\end{array}$ & $<1$ & $\begin{array}{l}\text { Splenectomy, } \\
\text { CHOP, CMOPP }\end{array}$ & Alive & 31.9 \\
\hline 8 & 33 & $\begin{array}{l}\text { Enlarged left } \\
\text { supraclavicular } \\
\text { lymph node }\end{array}$ & No & $3 \mathrm{~A}$ & $\mathrm{U}$ & CVP & $\begin{array}{l}\text { Yes } \\
\text { Grade 3A to } \\
\text { DLBCL }\end{array}$ & 5.6 & $\begin{array}{l}\text { BACOP, XRT, } \\
\text { BMT }\end{array}$ & DDU & 10.5 \\
\hline 9 & $\mathrm{U}$ & $\mathrm{U}$ & $\mathrm{U}$ & $3 \mathrm{~A}$ & IIIA & $\mathrm{U}$ & $\mathrm{U}$ & NA & $\mathrm{U}$ & Alive & 12.3 \\
\hline 10 & 64 & $\begin{array}{l}\text { Massive splenomegaly, } \\
\text { lymphadenopathy }\end{array}$ & No & $3 \mathrm{~A}$ & IIA & CHOP & $\begin{array}{l}\text { Yes } \\
\text { Grade 3A to } \\
\text { DLBCL }\end{array}$ & 1.1 & CEPP & DOD & 1.9 \\
\hline
\end{tabular}

U, unknown; +, positive; - , negative; R-CHOP, rituximab, cyclophosphamide, hydroxydaunomycin, vincristine sulfate, prednisone; CVP, cyclophosphamide, vincristine, prednisone; KLH, keyhole limpet hemocyanin; CMOPP, cyclophosphamide, vincristine, procarbazine, prednisone; XRT, radiation therapy; BMT, bone marrow transplant; BACOP, bleomycin, epidoxorubicin, cyclophosphamide, vincristine, and prednisone; CEPP, cyclophosphamide, etoposide, procarbazine, and prednisone; DOD, dead of disease; DDU, deceased, disease status unknown at time of death; NA, not applicable. 
EBV-positive follicular lymphoma, there were also six cases with bystander EBV-positive cells. These cases demonstrated scattered EBER-positive cells present in an interfollicular distribution that did not correspond to that of the lymphoma cells (Figure 4h). All of these were grade 1-2 follicular lymphoma. These cases were not considered to be EBV-positive follicular lymphoma.

\section{Discussion}

Previously reported cases of EBER positivity in association with follicular lymphoma are rare and are in the form of isolated case reports in patients with various immunodeficiency settings and usually in the context of transformation to diffuse large B-cell lymphoma and/or classical Hodgkin lymphoma. Koreishi et $a l^{6}$ identified a single case of a 65-year-old woman who was HIV-negative and was simultaneously found to have follicular lymphoma, Kaposi sarcoma and Castleman's disease with coinfection of EBV and HHV-8. In this case, the follicular lymphoma component was grade $3 \mathrm{~A}$ and large neoplastic centroblasts were positive for EBER. On the basis of the coexistence of follicular lymphoma, Kaposi sarcoma and of EBV and HHV-8, it is likely that this patient was significantly immunosuppressed, and thus this case report is distinct from our series in which history of immunosuppression and coexistence of other malignancies was not present in any of our EBV-positive cases. Orlandi et $\mathrm{al}^{7}$ also reported a single case of grade 1-2 follicular lymphoma arising in a 30-year-old male that transformed to a fatal EBV-related diffuse large B-cell lymphoma after treatment with multiple chemotherapy regimens, presumably due to the development of profound immunosuppression and uncontrolled reactivation of latent EBV. In that report, the EBV-associated lymphoma was classified as a diffuse large B-cell lymphoma, arising in the setting of iatrogenic immunosuppression. Menon et $a l^{8}$ reported a single case of grade 1-2 follicular lymphoma arising in a 53-year-old woman, which transformed to a grade $3 \mathrm{~A}$ follicular lymphoma, associated with CD30-positive, CD15-positive Hodgkin/Reed-Sternberg-like cells that were EBVpositive. Finally, although primary cutaneous follicle center lymphoma is considered to be a distinct entity from systemic or nodal based follicular lymphoma, it is notable to mention a case described by van der Horst et $a{ }^{10}$ of an EBV-positive primary cutaneous follicle center lymphoma, which arose in an immunocompetent 50-year-old man, who subsequently developed an EBV-positive diffuse large B-cell lymphoma.

In the current series, EBV was screened in a large cohort of unselected cases of typical follicular lymphoma from two academic institutions regardless of tumor grade, patient age, or immune status. In this report, we identified an overall prevalence of
$2.6 \%$ for EBV-positive follicular lymphoma. Six out of eight $(75 \%)$ of these cases also demonstrated coexpression of the EBV protein LMP1, indicating that EBV is present in latency type II or III in EBVpositive follicular lymphoma. Aside from advanced age and therefore possible age-related immunosenescence, and one patient with Sjögren's syndrome and treatment with hydroxychloroquine, there was no known history of immunodeficiency in these cases. To our knowledge, this is the first series reporting on EBV association in unselected follicular lymphomas occurring in patients with systemic or nodal based disease of any grade. The cases included in this study were collected from two academic institutions, thus we cannot exclude the possibility of bias in our cohorts based on referral practices to tertiary care and cancer centers.

In this cohort, EBV-positive follicular lymphoma was more frequently associated with grade $3 \mathrm{~A}-3 \mathrm{~B}$, having a prevalence rate of $10 \%$ among grade $3 \mathrm{~A}-3 \mathrm{~B}$ but only $0.4 \%$ in grade $1-2$ follicular lymphoma. EBV-positive follicular lymphoma was also significantly associated with CD30 coexpression. Despite the association with grade $3 \mathrm{~A}-3 \mathrm{~B}$ and CD30 expression, we did not identify any clinicopathologic features that could predict EBV status in unselected follicular lymphoma cases. Specifically, comparing tumors of the same grade, EBV-positive and EBVnegative cases of follicular lymphoma were otherwise morphologically and immunophenotypically indistinguishable, and unusual morphological or immunophenotypic features that may predict EBV association, such as Hodgkin-like features, necrosis, granulomas, angiocentric growth, or hemophagocytic lymphohistiocytosis were not identified in cases in this study. Although EBV was more prevalent among grade 3A-3B follicular lymphoma than among grade 1-2 follicular lymphoma, overall most grade 3A-3B cases were EBV-negative, and, therefore, the grade of the lymphoma likewise did not predict EBV status. Regarding patient age, we did not identify a statistically significant difference in age between EBV-positive and EBV-negative follicular lymphomas, thus any potential agerelated immunosenescence could have affected both EBV-positive and EBV-negative patient groups similarly and cannot be used to predict EBV positivity in these lymphomas.

Seven of the patients with EBV-positive follicular lymphoma in this cohort had additional (prior or subsequent) biopsies from different time points, all of which demonstrated progression of disease over time to higher-grade follicular lymphoma or diffuse large B-cell lymphoma. In one of the EBV-positive follicular lymphoma cases that progressed to EBVpositive diffuse large B-cell lymphoma, the initial biopsies of follicular lymphoma, which were excisional biopsies, lacked a diffuse large B-cell lymphoma component. This finding raises the question of the relationship between EBV-positive follicular lymphoma and EBV-positive diffuse large B-cell 
lymphoma. Previously, EBV-positive diffuse large B-cell lymphoma of the 'elderly' was considered in the 2008 WHO classification as a provisional entity ${ }^{11}$ and was defined as an EBV-related clonal large B-cell lymphoma that arises in elderly patients ( $>50$ years in age), without evidence of immunodeficiency or history of lymphoma. Initially, EBV-positive diffuse large B-cell lymphoma of the 'elderly' had shown a median age at diagnosis of 71 years, a slight male predominance (1.4:1), and was most often identified in cases with a non-germinal center origin. ${ }^{12,13}$ More recently, this entity has increasingly been reported in younger patients, including children, ${ }^{14}$ and the requirement for age $>50$ has been removed in the revised 2016 update to the WHO classification and these lymphomas are now classified as EBV-positive diffuse large B-cell lymphoma, NOS. ${ }^{15}$ These lymphomas were initially thought to arise as a de novo EBV-positive diffuse large B-cell lymphoma as opposed to arising in the setting of transformation of a pre-existing or coexisting EBV-positive follicular lymphoma. Originally considered a more aggressive variant, more recent studies of EBV-positive diffuse large B-cell lymphoma have shown variable results with regard to clinical outcome. ${ }^{16}$

Our series describing EBV-positive follicular lymphoma shares some similarities with EBVpositive diffuse large B-cell lymphoma, namely EBV positivity predominantly within large (highgrade) lymphoma cells, presence of EBV in latency type II or III, lack of morphological or immunophenotypic features that predict EBV positivity, prevalence of $2.6 \%$ in Western populations, and lack of history of immunosuppression. Also similar to current concepts in EBV-positive diffuse large B-cell lymphoma, the mean age of EBV-positive and -negative follicular lymphoma in this series did not differ significantly. In addition, in this series, EBER positivity was seen in association with CD30 expression by lymphoma cells. This is not surprising given the fact that CD30, a member of the tumor necrosis factor receptor superfamily, is a known activation induced antigen whose expression is upregulated in the setting of EBV infection in nonHodgkin lymphoma cells. ${ }^{17}$ Similar to the EBVpositive, CD30-positive follicular lymphomas described here, previous studies have shown that a high percentage of EBV-positive diffuse large B-cell lymphomas express CD30 (refs 13,18). The identification of EBV-positive follicular lymphoma and its tendency to progress to high-grade follicular lymphoma or EBV-positive diffuse large B-cell lymphoma raises the possibility that at least a subset of EBV-positive diffuse large B-cell lymphoma may arise in the context of progression from other EBVrelated lymphomas, such as follicular lymphoma. The clinicopathological similarities between EBVpositive follicular lymphoma and EBV-positive diffuse large B-cell lymphoma further support this possibility. These findings also raise questions regarding whether there is a prognostic or thera- peutic significance of identifying EBER and CD30 expression in follicular lymphoma, and whether identifying if an EBV-positive diffuse large B-cell lymphoma has arisen in the context of another EBVpositive lymphoma has clinical significance. These questions will need to be addressed in additional studies.

Similarly, in the context of follicular lymphoma, the $\mathrm{t}(14 ; 18)(\mathrm{q} 32 ; \mathrm{q} 21)$ translocation, found in $~ 85 \%$ of cases, results in the overexpression of the antiapoptotic BCL2 protein. However, this genetic event in isolation is not sufficient to induce follicular lymphoma. Instead, this translocation provides a survival advantage through activation of antiapoptotic programs that are typically repressed by BCL6 in germinal center $B$ cells. It is possible that EBV infection could result in additional genetic changes that contribute to transformation or progression of follicular lymphoma. There is emerging evidence indicating that EBV-encoded microRNAs can regulate expression of cellular messenger RNAs. These interactions have been shown to have important effects in the regulation of cellular pathways including those of cell survival and proliferation, and specifically p53, B-cell signaling, and cell proliferation and apoptosis pathways. ${ }^{19}$

It is unclear at this time whether EBV infection is an early event in the development of follicular lymphoma, thus participating in the genesis of the lymphoma, or whether EBV infection is a late event that may contribute to the progression of an already established lymphoma. In our experience, we have seen rare anecdotal cases of reactive follicular hyperplasias, in which reactive germinal centers contained EBV-positive cells as determined by in situ hybridization for EBER. In these cases, EBER positivity was focal, confined to few cells within few scattered reactive follicles. This is in contrast to the EBV-positive follicular lymphoma herein described, in which EBER positivity is generally diffuse among and within neoplastic follicles. However, it is important to mention that the number of EBV positive cells inside of an individual neoplastic follicle is variable, suggesting that not all of the neoplastic cells are infected by EBV. Also, we know that the vast majority of follicular lymphomas occur without EBV infection. On the basis of this observation, we believe that EBV infection may not be the driver event responsible for EBV-positive follicular lymphoma in most cases but instead is a facilitator of disease progression. For example, in $\mathrm{t}(14 ; 18)$ positive follicular lymphoma, if EBV infection of lymphoma cells predated the acquisition of $t(14 ; 18)$, then one would expect all clonally related follicular lymphoma cells to be EBV-positive, which is not the case in our experience. However, in our series we did identify one patient who had a low-grade follicular lymphoma, which was EBER-positive who then demonstrated progression to diffuse large B-cell lymphoma, EBV-positive, after 7.2 years (patient 5). The long interval between the initial EBV-positive 
follicular lymphoma and the subsequent diffuse large B-cell lymphoma in this single case suggests that EBV was present early on in follicular lymphomagenesis and may therefore be an early event in the genesis of EBV-positive follicular lymphoma, at least in a subset of cases. In future cases, the combination of fluorescent in situ hybridization for $\mathrm{t}(14 ; 18)$ with EBER (by in situ hybridization) will help to further assess this point. Theoretically, gene expression analysis of sorted follicular lymphoma cells (EBVpositive versus EBV-negative) in the same patient may be useful to better understand the contribution of EBV infection to lymphomagenesis and follicular lymphoma progression.

In summary, we present $10 \mathrm{EBV}$-positive cases in a large series of unselected patients with otherwise typical follicular lymphoma. The EBV-positive follicular lymphomas identified in this series had a prevalence of $2.6 \%$, contained EBV of latency type II or III, and most arose in immunocompetent patients. They were clinically, morphologically and immunophenotypically indistinguishable from EBVnegative cases of similar grade and therefore could only be identified by screening for EBV in all follicular lymphoma cases. EBV-positive follicular lymphoma shares some clinicopathological features with EBV-positive diffuse large B-cell lymphoma, such as association with grade $3 \mathrm{~A}-3 \mathrm{~B}$, diffuse EBER positivity within lymphoma cells ( $>75 \%$ of lymphoma cells in $90 \%$ of cases) and frequent strong coexpression of CD30. All cases in which biopsy material was available at separate time points demonstrated progression of disease to a higher-grade follicular lymphoma or to diffuse large B-cell lymphoma. This finding may have important implications regarding patient assessment in EBV-positive diffuse large B-cell lymphoma as well as possible therapeutic implications in low-grade tumors in which EBV is identified. Given that predictive or surrogate morphological or clinical features for EBV association in follicular lymphoma are unknown at this time, we believe that the only way to assess for the presence of EBV is by screening in all follicular lymphoma cases. Although the incidence of EBV in follicular lymphoma is low, it is higher in high-grade follicular lymphoma and for this reason we suggest that EBER should be screened for in all high-grade cases. Because the incidence of EBV is particularly low in low-grade follicular lymphoma, screening for EBER in all cases may not be cost effective. However, additional studies are needed to determine whether the presence of EBV is predictive of aggressive disease or if EBV-positive low-grade follicular lymphomas have a higher association with progression to highgrade follicular lymphoma or diffuse large B-cell lymphoma. If this is the case, then screening for EBV even in low-grade follicular lymphoma could be considered. Our findings expand the spectrum of recognized EBV-associated B-cell lymphomas. As this is an initial description, the true incidence of EBV association in follicular lymphoma, relationship to lymphomagenesis or transformation, and prognostic significance will need to be assessed in future studies.

\section{Acknowledgments}

This work has been supported in part by research funds from Sylvester Comprehensive Cancer Center (to FV) and from the Pathology Department (to JC).

\section{Disclosure/conflict of interest}

The authors declare no conflict of interest.

\section{References}

1 Geng L, Wang X. Epstein-Barr Virus-associated lymphoproliferative disorders: experimental and clinical developments. Int J Clin Exp Med 2015;8:14656-14671.

2 Cesarman E. Gammaherpesvirus and lymphoproliferative disorders in immunocompromised patients. Cancer Lett 2011;305:163-174.

3 Teramoto N, Sarker AB, Tonoyama Y, et al. EpsteinBarr virus infection in the neoplastic and nonneoplastic cells of lymphoid malignancies. Cancer 1996;77: 2339-2347.

4 Thorley-Lawson DA, Gross A. Persistence of the Epstein-Barr virus and the origins of associated lymphomas. N Engl J Med 2004;350:1328-1337.

5 Stein H, Warnke RA, Chan WC, et al. Diffuse large B-cell lymphoma, not otherwise specified. In: Swerdlow SH, Campo E, Harris NL, et al. (eds). WHO Classification of Tumours of Haematopoetic and Lymphoid Tissues, 4th edn. International Agency for Research on Cancer (IARC): Lyon, France, 2008, pp 233-261.

6 Koreishi AF, Saenz AJ, Arcila ME, et al. Synchronous follicular lymphoma, kaposi sarcoma, and castleman's disease in a HIV-negative patient with EBV and HHV-8 coinfection. Int J Surg Pathol 2011;19:685-691.

7 Orlandi E, Paulli M, Viglio A, et al. Epstein-Barr virus-positive aggressive lymphoma as consequence of immunosuppression after multiple salvage treatments for follicular lymphoma. Br J Haematol 2001;112: 373-376.

8 Menon MP, Hutchinson L, Garver J, et al. Transformation of follicular lymphoma to Epstein-Barr virusrelated Hodgkin-like lymphoma. J Clin Oncol 2013;31: e53-e56.

9 Dojcinov SD, Venkataraman G, Pittaluga S, et al. Agerelated EBV-associated lymphoproliferative disorders in the Western population: a spectrum of reactive lymphoid hyperplasia and lymphoma. Blood 2011;117: 4726-4735.

10 van der Horst MP, Hardwick A, Rahilly M, et al. Epstein-Barr virus-positive primary cutaneous follicle centre lymphoma; an age-related phenomenon? Virchows Arch 2015;467:111-117.

11 Nakamura S, Jaffe ES, Swerdlow SH, et al. EBV positive diffuse large B-cell lymphoma of the elderly. In: Swerdlow $\mathrm{SH}$, Campo E, Harris NL, et al. (eds). WHO Classification of Tumours of Haematopoetic and Lymphoid Tissues, 4th edn. International Agency for Research on Cancer (IARC): Lyon, France, 2008, pp 243-244. 
12 Gibson SE, Hsi ED. Epstein-Barr virus-positive B-cell lymphoma of the elderly at a United States tertiary medical center: an uncommon aggressive lymphoma with a nongerminal center B-cell phenotype. Hum Pathol 2009;40:653-661.

13 Montes-Moreno S, Odqvist L, Diaz-Perez JA, et al. EBVpositive diffuse large B-cell lymphoma of the elderly is an aggressive post-germinal center B-cell neoplasm characterized by prominent nuclear factor-kB activation. Modern Pathol 2012;25:968-982.

14 Nicolae A, Pittaluga S, Abdullah S, et al. EBV-positive large B-cell lymphomas in young patients: a nodal lymphoma with evidence for a tolerogenic immune environment. Blood 2015;126:863-872.

15 Swerdlow SH, Campo E, Pileri SA, et al. The 2016 revision of the World Health Organization classification of lymphoid neoplasms. Blood 2016;127: 2375-2390.

16 Ok CY, Li L, Xu-Monette ZY, et al. Prevalence and clinical implications of epstein-barr virus infection in de novo diffuse large B-cell lymphoma in Western countries. Clin Cancer Res 2014;20:2338-2349.

17 Kanavaros P, Jiwa NM, de Bruin PC, et al. High incidence of EBV genome in CD30-positive non-Hodgkin's lymphomas. J Pathol 1992;168:307-315.

18 Slack GW, Steidl C, Sehn LH, et al. CD30 expression in de novo diffuse large B-cell lymphoma: a populationbased study from British Columbia. Br J Haematol 2014;167:608-617.

19 Ma J, Nie K, Redmond D, et al. EBV-miR-BHRF1-2 targets PRDM1/Blimp1: potential role in EBV lymphomagenesis. Leukemia 2016;30:594-604. 